\title{
Correlación entre score de calcio coronario, esteatosis hepática y síndrome metabólico
}

Dres. Karina Hermosilla $M^{(1)}$, Daniela Pivcevic $C^{(2)}$, Julia Alegría $B^{(1)}$, Claudio Silva $F^{(1)}$.

1. Médico Radiólogo. Facultad de Medicina Clínica Alemana de Santiago. Universidad del Desarrollo. Santiago, Chile.

2. Becado de Radiología, Facultad de Medicina Clínica Alemana de Santiago. Universidad del Desarrollo. Santiago, Chile.

\section{Correlation between coronary calcium score, hepatic steatosis and metabolic syndrome}

\begin{abstract}
Objectives. To demonstrate the correlation between the presence or absence of established coronary disease (as measured by the Agatston index) and hepatic steatosis visualized in liver segments when calculating calcium score. Material and Methods. Retrospective study that included 229 patients with risk factors for coronary disease who underwent multislice computed tomography of coronary arteries or calcium score. We evaluated the presence of atherosclerotic disease in coronary arteries and thoracic aorta and liver attenuation in visualized liver segments. Statistical analysis included linear regression models, association studies of multiple variables and CART model. Results. 229 patients, $78 \%$ male, average age 56 years. It values statistically significant association was found between higher levels of calcium score and lower hepatic attenuation in older patients and males. We were to define two types of patients with high calcium score, a group with hypertension - hepatic steatosis and another groups with hypertension and type 2 diabetes; patients that were only obese did not have higher levels of calcium score. Conclusions. A relationship exists between calcified atheromatosis disease and hepatics steatosis. Patients with hepatics steatosis as part of a metabolic syndrome are at increased risk of atherosclerosis. It may be useful to incorporate the assessment of hepatics steatosis in cardiovascular risk stratification. Keywords: Atheromatosis, Calcium score, Hepatic steatosis, Metabolic syndrome.
\end{abstract}

Resumen: Objetivos. Demostrar la correlación que existe entre presencia o no de enfermedad coronaria establecida (medida a través del índice de Agatston) y esteatosis hepática en los segmentos visualizados del hígado al momento de realizar el score de calcio. Material y Métodos. Estudio retrospectivo que incluyó 229 pacientes con factores de riesgo para enfermedad coronaria a los que se les realizó tomografía computada multicorte de arterias coronarias o score de calcio. Se evaluó la presencia de enfermedad ateromatosa en arterias coronarias y aorta torácica y atenuación hepática en los segmentos visualizados del hígado. El análisis estadístico incluyó modelos de regresión lineal, estudio por asociación de múltiples variables y modelo CART. Resultados. 229 pacientes, $78 \%$ de sexo masculino y 22\% femenino, con edad promedio 56 años. Se demostró asociación estadísticamente significativa entre mayor nivel de score de calcio y menor atenuación hepática, en pacientes de mayor edad y sexo masculino. Se logró definir dos tipos de pacientes con score de calcio elevado, un grupo con hipertensión arterial - esteatosis hepática y otro grupo con hipertensión arterial y diabetes tipo 2; los pacientes solamente obesos no tenían mayores niveles de score de calcio. Conclusiones. Existe asociación entre ateromatosis calcificada y esteatosis hepática. Los pacientes con esteatosis hepática como parte de un síndrome metabólico, tienen mayor riesgo de ateroesclerosis. Puede ser útil incorporar la evaluación de esteatosis hepática en la estratificación de riesgo cardiovascular.

Palabras clave: Ateromatosis, Esteatosis, Hepática, Score de calcio, Síndrome metabólico.

Hermosilla K, et al. Correlación entre score de calcio coronario, esteatosis hepática y síndrome metabólico. Rev Chil Radiol 2013; 19(1): 64-68.

Correspondencia: Dra. Karina Hermosilla / karihermosilla@gmail.com

Trabajo recibido el 23 de enero de 2013, aceptado para publicación el 22 de febrero de 2013.

\section{Introducción}

El hígado graso no alcohólico comprende un espectro de condiciones patológicas, que incluyen la esteatosis simple, esteato-hepatitis no alcohólica y cirrosis. Esta condición ha alcanzado proporciones de epidemia y es la causa más común de enfermedad hepática crónica ${ }^{(1)}$.

En países occidentales se estima que aproximadamente $20-30 \%$ de los adultos en la población general, 
tienen hígado graso no alcohólico, y su prevalencia aumenta de 70 - 90\% entre las personas obesas o con diabetes; estos pacientes también presentan aumento del riesgo para el desarrollo de cirrosis ${ }^{(4)}$.

La prevalencia de hígado graso no alcohólico se espera que aumente debido a los hábitos dietéticos y estilo de vida cada vez más sedentario. La biopsia hepática es el estándar de oro para establecer el diagnóstico de hígado graso no alcohólico, pero es invasiva y puede presentar complicaciones como hemorragia. La tomografía computada multicorte (TCMC) se puede utilizar para diagnosticar hígado graso, mediante la demostración de baja atenuación en el parénquima hepático (82\% de sensibilidad y $100 \%$ de especificidad) ${ }^{(2)}$.

La esteatosis hepática está estrechamente relacionada con varios trastornos metabólicos, como la resistencia a la insulina y se ha propuesto como el factor unificador de los trastornos metabólicos.

La enfermedad arterial coronaria (EAC) también está relacionada con varios trastornos metabólicos, lo que aumenta la preocupación de que los pacientes con hígado graso no alcohólico también podrían estar en mayor riesgo de enfermedad coronaria.

En la práctica cardiológica la TCMC es un método no invasivo de diagnóstico de enfermedad coronaria en
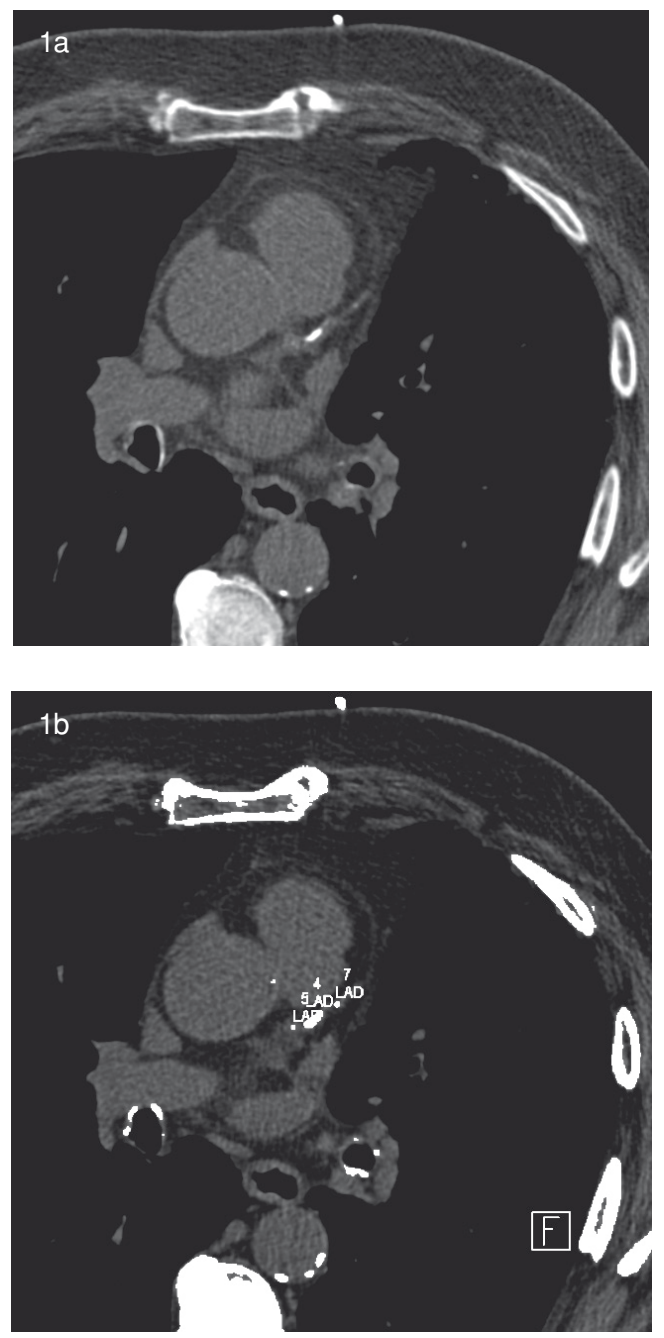

pacientes con factores de riesgo cardiovascular leve o moderado $^{(3)}$.

Dado que los pacientes esteatosis hepática tiene alto riesgo de desarrollar diabetes y enfermedades cardiovasculares $^{(4)}$, con este trabajo pretendemos demostrar la correlación que existe entre presencia o no de enfermedad coronaria establecida (medida a través del índice de Agatston) y esteatosis hepática en los segmentos visualizados del hígado al momento de realizar el score de calcio ${ }^{(5)}$.

\section{Material y métodos}

Estudio retrospectivo que incluyó 229 pacientes con factores de riesgo de enfermedad coronaria, que se realizaron TCMC de arterias coronarias o score de calcio en el Departamento de Tomografía Computada y Resonancia Magnética de la Clínica Alemana de Santiago entre octubre de 2009 y octubre de 2010 . Se excluyeron pacientes con by-pass coronario.

Todos los exámenes fueron realizados en un tomógrafo multidetector de 128 canales, con adquisión prospectiva, gatillada por electrocardiograma, sin uso de medio de contraste ev, o previo al uso de este en el caso de TCMC de arterias coronarias (Figuras 1a-c). Los exámenes fueron revisados por dos radiólogos especialistas en imágenes cardiotorácicas.

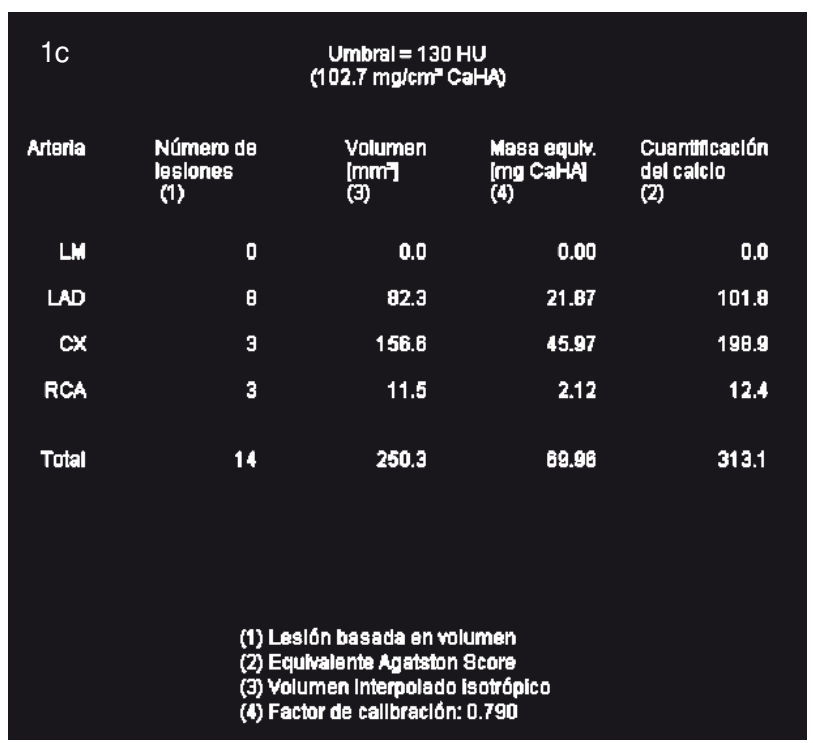

Figura 1a-c. Determinación de Score de calcio por TCMC. El software semi-automático identifica aquellos pixeles sobre el umbral de $130 \mathrm{UH}$ (Figuras 1a y 1b), los cuales son asignados manualmente a una de las ramas coronarias, arrojando el valor final de cuantificación de calcio (Figura 1c). 
Se clasificaron los pacientes según la presencia de enfermedad ateromatosa calcificada y no calcificada en arterias coronarias y aorta torácica.

Se midió atenuación del parénquima hepático en los segmentos visualizados del hígado al momento de realizar el score de calcio en Unidades Hounsfield (UH); esto fue realizado aplicando un ROI de al menos $5 \mathrm{~cm}^{2}$ sobre un segmento del lóbulo hepático izquierdo (Figura 2).

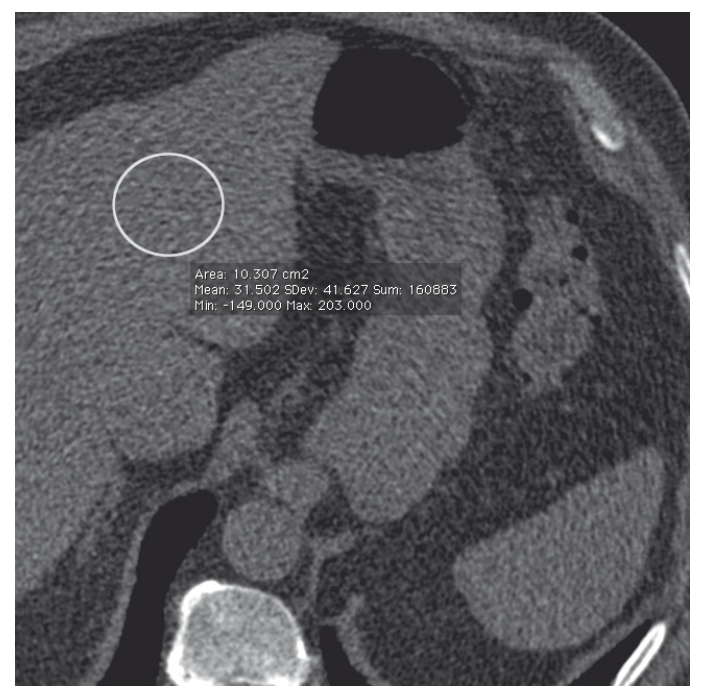

Figura 2. Medición de atenuación del parénquima hepático en los segmentos visualizados del hígado al momento de realizar el score de calcio en unidades Hounsfield(UH).

Se revisó la ficha clínica electrónica de los 225 pacientes seleccionados obteniéndose los siguientes datos: sexo, edad, presencia de hipertensión arterial (HTA), diabetes mellitus tipo 2 (DM), dislipidemia, obesidad e hígado graso no alcohólico. Se excluyeron 4 pacientes que no tenían registro completo en la ficha clínica.

Para el análisis estadístico se usaron modelos de regresión lineal, estudio por asociación de múltiples variables y modelo CART. Se utilizó programa
Tabla I. Características pacientes.

Pacientes
Edad (años)
Hombres
Mujeres
Diabetes
Hipertensión
Dislipidemia

221
56 (DS: 11)
172
49
21
90
139

$77,8 \%$

$22,2 \%$

$9,50 \%$

$40,72 \%$

$62,90 \%$

estadístico STATA v10.1 (STATA Statistical Software: Release 10. College Station, TX: StataCorp LP). Se consideró $p<0.05$ como diferencias estadísticamente significativas.

\section{Resultados}

La población de estudio consistió en 221 pacientes, 172 hombres (78\%) y 49 mujeres (22\%) con un promedio de edad de 56 años (+/- 11 D.S). De ellos, el $9,5 \%$ eran diabéticos, $40,72 \%$ hipertensos, y $62,9 \%$ dislipidémicos (Tabla I).

A través de análisis uni y multivariados se estableció que no hay diferencias significativas en la distribución de valores de atenuación hepática y valores de score calcio evaluados por sexo.

Si existe asociación estadísticamente significativa entre mayores valores de score de calcio con menores valores de atenuación hepática $(p=0,04)$, mayor edad $(p<0,001)$ y sexo masculino $(p=0,005)$ (Figura 3$)$.

No hay mayor asociación de ateromatosis no calcificada en relación a esteatosis (ni como variable continua ni como categórica). Sin embargo, si se demuestra una asociación estadísticamente significativa entre la presencia de ateromatosis calcificada y esteatosis hepática.

Aplicando un modelo de regresión lineal se estudio la relación entre los valores de score de calcio (como variable dependiente) y los valores de atenuación del parénquima hepático (UH), edad de los pacientes, sexo, HTA, DM, obesidad y dislipidemia; retirándose

\begin{tabular}{|lrrrrrr|}
\hline Score Calcio & Coef. & SE & $\mathbf{t}$ & P >t & \multicolumn{2}{c|}{ [Intervalo confianza 95\%] } \\
\hline UH & 3,105 & 1,574 & 1.97 & 0.040 & 0.003 & 6,208 \\
Edad & 12,158 & 1,834 & 6.63 & 0.000 & 8,543 & 15,774 \\
Sexo & $-139,981$ & 49,162 & -2.85 & 0.005 & $-236,888$ & $-43,074$ \\
Diabetes & $-104,119$ & 75,197 & -1.38 & 0.168 & $-252,346$ & 44,107 \\
Displipidemia & 42,857 & 43,425 & 0.99 & 0.325 & $-42,742$ & 128,457 \\
Obesidad & 4,830 & 54,622 & 0.09 & 0.930 & $-102,839$ & 112,501 \\
HTA & 68,355 & 44,628 & 1.53 & 0.127 & $-19,614$ & 156,325 \\
& & & & & & \\
\hline
\end{tabular}

Figura 3. Coeficientes de regresión que muestra asociación estadísticamente significativa entre mayores valores de score de calcio y la presencia de menor valor atenuación del parénquima hepático, mayor edad y sexo masculino. 


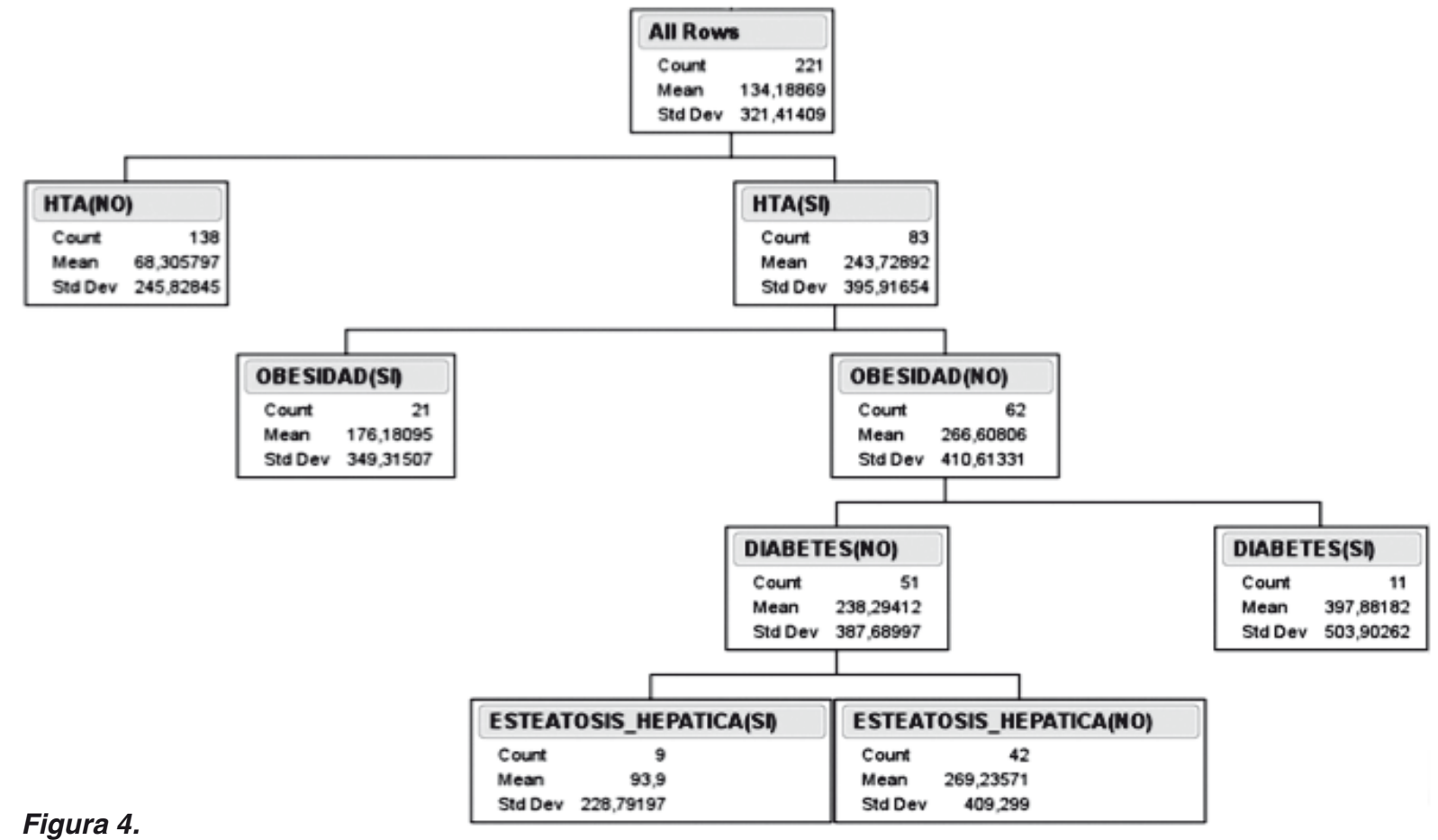

los valores no significativos del modelo, obteniéndose la siguiente ecuación final:

Score $=-511-156$ * sexo +13.1 * edad +2.89 * UH hepático

Demostrando que los pacientes con menores niveles de atenuación hepática y mayor edad, presentan mayores niveles de score de calcio.

La técnica CART se basa en que las combinaciones de factores de riesgos para una patología son únicas para un paciente en particular, pero tienen un número limitado de combinaciones en la población. Por eso, cuando se tiene un número grande de pacientes y variables, se puede ver cuales de estas combinaciones concentran los pacientes de mayor riesgo de enfermedad (Figura 4).

Así en éste caso se pudo establecer dos grupos de pacientes con los mayores niveles de score de calcio (que presentan mayor riesgo coronario): Un grupo con HTA y esteatosis hepática y un segundo grupo con HTA y DM tipo 2. (Figura 5).

Los pacientes que presentaban solamente obesidad no expresaban mayores niveles de score de calcio.

\section{Discusión}

El índice (score) de Agatston, expresa de forma general la magnitud de calcio depositado en el árbol

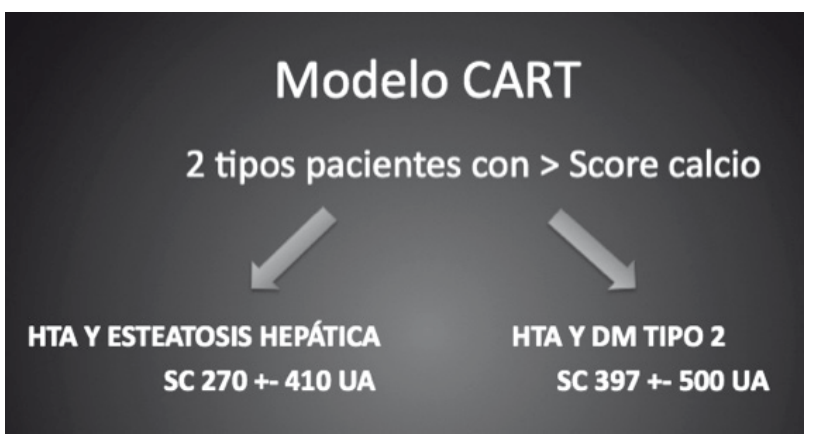

Figura 5.

coronario, estableciendo un umbral de densidad en UH y su expresión en masa adyacente. El calcio como expresión sustituta de la carga aterosclerótica coronaria del sujeto, es de gran relevancia epidemiológica y pronóstica. Así, Rumberger, et a/(6) demuestran que un score de calcio coronario (SCC) superior a 371 tiene un $90 \%$ de especificidad para al menos una lesión coronaria con estenosis superior al $70 \%$. Los score de calcio coronario superiores a 1.000 son los que tienen mayor probabilidad de síndromes coronarios agudos. El estudio MESA (Multi - Ethnic Study of Atherosclerosis) ha demostrado que modelamientos que incluyen raza, sexo y edad asociado al valor absoluto de score de calcio, son predictores de eventos adversos ${ }^{(7)}$.

Con este trabajo se logró demostrar que existe una asociación estadísticamente significativa en 
el grupo de pacientes estudiados, entre la presencia de ateromatosis calcificada coronaria y la presencia esteatosis hepática, probablemente esto sea secundario a que la esteatosis hepática se presenta en síndromes metabólicos de mayor tiempo evolución.

Dado que los pacientes con esteatosis hepática, como parte de un síndrome metabólico, tienen mayor riesgo de ateroesclerosis, puede ser útil incorporar la evaluación de esteatosis hepática en la estratificación de riesgo cardiovascular, considerando además que esta información esta disponible en la adquisición estándar de las tomografías computadas de arterias coronarias y mediciones de score de calcio, además no aumenta los tiempo de adquisión del examen ni la exposición a radiación ionizante.

\section{Bibliografía}

1. Targher G, Day C, Bonora E. Risk of Cardiovascular Disease in Patients with Nonalcoholic Fatty Liver Disease. N Engl J Med 2010; 363: 1341-1350.

2. Bohte AE, van Werven JR, Bipat S, Stoker J. The diagnostic accuracy of US, CT, MRI and $1 \mathrm{H}-\mathrm{MRS}$ for the evaluation of hepatic steatosis compared with liver biopsy: a meta-analysis. Eur Radiol 2011 Jan; 21(1): 87-97.

3. Taylor AJ, Cerqueira M, Hodgson JM, Mark D, Min J,
O'Gara P, et al. ACCF/SCCT/ACR/AHA/ASE/ASNC/ NASCI/SCAI/SCMR 2010 appropriate use criteria for cardiac computed tomography. A report of the American College of Cardiology Foundation Appropriate Use Criteria Task Force, the Society of Cardiovascular Computed Tomography, the American College of Radiology, the American Heart Association, the American Society of Echocardiography, the American Society of Nuclear Cardiology, the North American Society for Cardiovascular Imaging, the Society for Cardiovascular Angiography and Interventions, and the Society for Cardiovascular Magnetic Resonance. J Am Coll Cardiol 2010 Nov 23; 56(22): 1864-1894.

4. Chen CH, Nien CK, Yang CC, Yeh YH. Association Between Nonalcoholic Fatty Liver Disease and Coronary Artery Calcification. Dig Dis Sci 2010; 55: 1752-1760.

5. Gastaldelli A, Kozakova M, Hojlund K, et al. Fatty liver is associated with insulin resistance, risk of coronary heart isease, and early atherosclerosis in a large European population. Hepatology 2009; 49: 1537-1544.

6. Rumberger JA, Brundage BH, Rader DJ, Kondos G. Electron beam computed tomographic coronary calcium scanning: a review and guidelines for use in asymptomatic persons. Mayo Clin Proc 1999; 74: 243-252.

7. Budoff MJ, McClelland RL, Nasir K, et al. Cardiovascular events with absent or minimal coronary calcification: the Multi-Ethnic Study of Atherosclerosis (MESA). Am Heart J 2009 Oct; 158 (4): 554-561. 